\title{
Checkpoint kinase-1 inhibition and etoposide exhibit a strong synergistic anticancer effect on chronic myeloid leukemia cell line K562 by impairing homologous recombination DNA damage repair
}

\author{
ZHUOYI FAN $^{1}$, HUACHENG LUO ${ }^{2}$, JIE ZHOU ${ }^{1}$, FANGCE WANG ${ }^{1}$, WENJUN ZHANG ${ }^{1}$, JIAN WANG $^{3}$, \\ SHUO LI $^{4}$, QIAN LAI ${ }^{5}$, YUESHUANG XU ${ }^{6}$, GUANGMING WANG ${ }^{1,3}$, AIBIN LIANG $^{1}$ and JUN XU ${ }^{3}$ \\ ${ }^{1}$ Department of Hematology, Tongji Hospital, Tongji University School of Medicine, Shanghai 200065, P.R. China; \\ ${ }^{2}$ Division of Pediatric Hematology/Oncology, Department of Pediatrics, Pennsylvania State University College of Medicine, \\ Hershey, PA 17033, USA; ${ }^{3}$ East Hospital, Tongji University School of Medicine, Shanghai 200120, P.R. China; \\ ${ }^{4}$ Jake Gittlen Laboratories for Cancer Research, Pennsylvania State University College of Medicine, Hershey, \\ PA 17033, USA; ${ }^{5}$ Department of Hematology, The First Affiliated Hospital of Xiamen University and \\ Institute of Hematology, School of Medicine, Xiamen University, Xiamen, Fujian 361003, P.R. China; \\ ${ }^{6}$ Department of Pathology, School of Medicine, Southeast University, Nanjing, Jiangsu 210009, P.R. China
}

Received December 31, 2019; Accepted August 7, 2020

DOI: $10.3892 / o r .2020 .7757$

\begin{abstract}
Leukemia, a malignant hematological disease, has poor therapeutic outcomes due to chemotherapeutic resistance. Increasing evidence has confirmed that the elevated capacity for DNA damage repair in cancer cells is a major mechanism of acquired chemotherapeutic resistance. Thus, combining chemotherapy with inhibitors of DNA damage repair pathways is potentially an ideal strategy for treating leukemia. Checkpoint kinase $1(\mathrm{CHKl})$ is an important component of the DNA damage response (DDR) and is involved in the $\mathrm{G}_{2} / \mathrm{M}$ DNA damage checkpoint. In the present study, we demonstrated that shRNA-mediated CHK1 silencing suppressed cell proliferation and enhanced the cytotoxic effects of etoposide (VP16) in the chronic myeloid leukemia (CML) cell line K562 through the results of CCK-8, and comet assay. The results demonstrated that shRNA-induced CHK1 silencing can override $\mathrm{G}_{2} / \mathrm{M}$ arrest and impair homologous recombination (HR) repair by reducing breast cancer susceptibility gene 1 (BRCA1) expression. Cells had no time, and thus limited ability, to repair the damage and were thus more sensitive to chemotherapy
\end{abstract}

Correspondence to: Professor Aibin Liang, Department of Hematology, Tongji Hospital, Tongji University School of Medicine, Shanghai 200065, P.R. China

E-mail: lab7182@tongji.edu.cn

Professor Jun Xu, East Hospital, Tongji University School of Medicine, Shanghai 200120, P.R. China

E-mail: xunymc2000@yahoo.com

Key words: CHK1, CCT245737, DNA damage response, etoposide, chronic myeloid leukemia after CHK1 downregulation. Second, we tested the therapeutic effect of VP16 combined with CCT245737, an orally bioavailable CHK1 inhibitor, and observed strong synergistic anticancer effects in K562 cells. Moreover, we discovered that CCT245737 significantly prevented the $\mathrm{G}_{2} / \mathrm{M}$ arrest caused by acute exposure to VP16. Interestingly, CCT245737 inhibited both BRCA1 and Rad51, the most important component of the HR repair pathway. In conclusion, these results revealed that CHK1 is potentially an ideal therapeutic target for the treatment of CML and that CCT245737 should be considered a candidate drug.

\section{Introduction}

Although tyrosine kinase inhibitors (TKIs) have revolutionized the treatment of chronic myeloid leukemia (CML), some CML patients experience TKI resistance $(1,2)$, TKI intolerance (3) or economic plight (4). Thus, there is still a need to identify new approaches for CML treatment.

Despite the vast improvements in therapeutic strategies, DNA damage-based chemotherapy agents currently remain the preferred choice to treat most leukemias. DNA damage can induce cell cycle arrest and activate repair pathways, which are the main mechanisms of resistance to these preferred chemotherapy drugs. Therefore, combining DNA damage response (DDR) pathway inhibitors with DNA damage-based chemotherapeutics is a potential new strategy for treating leukemia, including CML.

In general, DNA damage induces cell cycle arrest by activating cell cycle checkpoints, which gives cells enough time to repair the damage. Cancer cells mostly rely on the $\mathrm{G}_{2} / \mathrm{M}$ checkpoint to repair damaged DNA due to inactivating mutations or loss of p53 $(5,6)$. Thus, we were inspired to target important components of the $\mathrm{G}_{2} / \mathrm{M}$ checkpoint to treat cancer, 
especially p53-mutant cancer. Two major signaling pathways are involved in the DDR: ATM/checkpoint kinase 2 (CHK2) and ATR/CHK1 (7-9). Research has shown that in malignant cells, the components involved in the ATR/CHK1 pathway are often upregulated, while ATM/CHK2 components are often deficient (10). Hence, the ATR/CHK1 signaling pathway, which is also involved in the $\mathrm{G}_{2} / \mathrm{M}$ checkpoint, is a potential target for the treatment of cancer.

CHK1 is a Ser/Thr protein kinase that was first discovered in fission yeast in 1993 (11). In many different cancers, resistance to chemotherapy and radiotherapy is associated with CHK1 overexpression. CHK1 is phosphorylated by ATR in response to DNA damage and subsequently phosphorylates its downstream substrates to induce cell cycle arrest (12-14). Subsequent research confirmed that both the $\mathrm{G}_{2} / \mathrm{M}$ checkpoint and $\mathrm{CHK} 1$ play critical roles in the $\mathrm{S}$ phase and mitotic checkpoints $(15,16)$. The CHK1 pathway can directly facilitate homologous recombination (HR) by regulating the expression, activity or localization of related proteins $(17,18)$. Therefore, CHK1 is an attractive candidate for anticancer therapy.

To date, several CHK1 inhibitors have been developed and administered either alone or in combination with other chemotherapeutic agents in preclinical or clinical trials (19-25). Among them, CCT245737 ((R)-5-((4-((morpho lin-2-ylmethyl)amino)-5-(trifluoromethyl)pyridin-2-yl)amino) pyrazine-2-carbonitrile) is a selective CHK1 inhibitor that has oral bioavailability $(26,27)$. Previous research has shown that CCT245737 can enhance the anticancer effects of gemcitabine and carboplatin in non-small cell lung cancer (NSCLC) and B-cell lymphoma mouse models (26). However, no study has tested the potential of this inhibitor to increase the efficacy of chemotherapy or elucidated the mechanism by which this inhibitor enhances chemotherapeutic effects in CML.

In the present study, CHK1 was inhibited by RNA interference (RNAi) or CCT245737 in K562 cells to determine its role in the chemosensitivity of CML cells. We confirmed that CHK1 inhibition significantly and specifically enhanced the chemosensitivity of K562 cells to etoposide (VP16) by eliminating VP16-induced $\mathrm{G}_{2} / \mathrm{M}$ arrest and suppressing HR efficiency. Moreover, we also preliminarily investigated the therapeutic effect of CCT245737 in imatinib-treated K562 cells and showed that this approach could be applicable to TKI treatment of CML in the future.

\section{Materials and methods}

Cell lines. K562 (human CML) and 293T/17 cells were purchased from the Cell Bank, Chinese Academy of Sciences (Shanghai, China); the KCL22 (human CML) cell line was obtained from the German Collection of Cell Cultures (Braunschweig, Germany); and the CAM191 (human normal lymphocyte) cells were purchased from the Cell Bank, Chinese Academy of Sciences (Kunming, China). K562 and KCL22 cells were grown in IMDM (Gibco; Thermo Fisher Scientific, Inc.), CAM191 cells were grown in RPMI-1640 medium (Gibco; Thermo Fisher Scientific, Inc.), and 293T/17 cells were grown in DMEM (Gibco; Thermo Fisher Scientific, Inc.). All media were supplemented with $100 \mathrm{U} / \mathrm{ml}$ penicillin, $0.1 \mathrm{mg} / \mathrm{ml}$ streptomycin and $10 \%$ fetal bovine serum (FBS; Gibco; Thermo Fisher Scientific, Inc.). The cells were incubated at $37^{\circ} \mathrm{C}$ in a humidified atmosphere containing $5 \% \mathrm{CO}_{2}$ and were used for experiments in the logarithmic growth phase.

Reagents. VP16, imatinib and the CHK1 inhibitor CCT245737 were obtained from Selleckchem (USA) and dissolved in DMSO (Sigma-Aldrich; Merck KGaA) for the in vitro studies. In the shRNA-mediated CHK1 knockdown study, cells were seeded at $10^{5} / \mathrm{ml}$ and then treated with $5 \mu \mathrm{M}$ VP16 for $4.5 \mathrm{~h}$ to induce DNA double-strand breaks (DSBs). After incubation, the cells were washed with PBS and maintained in IMDM for $48 \mathrm{~h}$ to allow the repair of VP16-induced DSBs before further analysis $(28,29)$. In the imatinib experiments, K562 cells were seeded at $10^{5} / \mathrm{ml}$ and then treated with $1 \mu \mathrm{M}$ imatinib for $24 \mathrm{~h}$. After incubation, live cells were acquired by washing with PBS and incubated for another $24 \mathrm{~h}$, and then, the cells were collected for subsequent experiments. In most CCT245737-induced CHK1 inhibition studies, in order to induce sustained DNA damage in cells and detect the role of CCT245737 on CHK1 activation more efficiently and to promote this pharmacological combination approach in the clinic more conveniently in the future, cells were treated with VP16 $(5 \mu \mathrm{M})$ and CCT245737 $(500 \mathrm{nM})$ either alone or in combination at the density of $10^{5} / \mathrm{ml}$ for $24 \mathrm{~h}$ as in previous research (22). Before further analysis, cells were washed with PBS. Primary antibodies against pSer296 CHK1 (cat. no. 2349), pSer317 CHK1 (cat. no. 2344), pSer345 CHK1 (cat. no. 2341), total CHK1 (cat. no. 2360), total H2AX (cat. no. 7631), pSer139 H2AX (cat. no. 9718), cleaved-PARP (cat. no. 5625), total H3 (cat. no. 4499), pSer10 H3 (cat. no. 53348), pSer216 CDC25c (cat. no. 4901), total CDK1 (cat. no. 9116), pTyr15CDK1 (cat. no. 4539), Rad51 (cat. no. 8875), Rad50 (cat. no. 3427), BRCA1 (cat. no. 9010) and GAPDH (cat. no. 2118) were purchased from Cell Signaling Technology, and antibodies against total CDC25c (product code 32444), MRE11 (product code 208020), Ku70 (product code 92450), Ku80 (product code 80592) and Lig4 (product code 193353) were purchased from Abcam.

RNAi-mediated gene knockdown. The shRNA sequence GCA ACAGTATTTCGGTATAAT was used to knock down CHK1 (shCHK1-KD), whereas the sequence GCGCGCTTTGTA GGATTCG, which is unrelated to any sequence in humans, served as a negative control (shRNA-NC). The pLVX-shRNA vector system containing either a puromycin resistance cassette or ZsGreen (Clontech Laboratories) was used to carry these sequences. In addition to shRNA-NC, the empty pLVX-shRNA vector was also used as a control (shRNA-Vector). To produce lentiviral particles, all of these plasmids (with two packaging plasmids at a ratio of 4:3:2) were cotransfected into 293T/17 cells via the calcium phosphate precipitation method. Viral infection was performed with $10 \mu \mathrm{g} / \mathrm{ml}$ polybrene, and GFP expression in cells was observed by a fluorescence microscope after 48-72 h. After positive cells were sorted, qPCR and western blotting were performed to detect the knockdown efficiency. Cells with significant target gene knockdown were used for subsequent experiments.

$R N A$ extraction and $q P C R$. Total RNA was extracted with a MiniBEST Universal RNA Extraction Kit (Takara), and cDNA was produced by the PrimeScript RT Master Mix (Takara). The TB Green Premix Ex Taq ${ }^{\mathrm{TM}}$ II (Tli RNaseH Plus) Kit (Takara) 
was used to detect relative mRNA expression by LightCycler 96 system (Roche) with 40 cycles of PCR thermocycling. The primer sequences were as follows: $\beta$-actin forward, 5'-GGA TTCCTATGTGGGCGACGA-3' and reverse, 5'-GCGTAC AGGGATAGCAC AGC-3'; CHK1 forward, 5'-CCAGATGCT CAGAGATTCTTCCA-3' and reverse, 5'-TGTTCAACAAAC GCTCACGATTA-3'. The mRNA expression of the target gene relative to $\beta$-actin expression was calculated by the $2^{-\Delta \Delta \mathrm{Cq}}$ method (30).

Protein extraction and western blotting. Cells were harvested and lysed in RIPA lysis buffer (Thermo Fisher Scientific, Inc.) containing protease and phosphatase inhibitors (Epizyme Scientific, China). Lysates were centrifuged to obtain protein extracts for western blotting. The BCA method (Beyotime Institute of Biotechnology) was used to detect the protein concentration. SDS-PAGE gels (10\% gel) (Epizyme, China) were used for protein electrophoresis (20-40 $\mu \mathrm{g}$ per sample), and the separated proteins were transferred to PVDF membranes (Millipore). Next, the membranes were blocked in $5 \%$ nonfat milk for $2 \mathrm{~h}$ at room temperature and washed 3 times with TBS-T. Then, the membranes were incubated with specific primary antibodies at the dilution of 1:1,000, at $4^{\circ} \mathrm{C}$ overnight. On the next day, the membranes were washed and then incubated for $2 \mathrm{~h}$ at room temperature with HRP-conjugated anti-rabbit or anti-mouse secondary antibodies at the dilution of 1:1,000 (cat. nos. 7074 and 7076; both from Cell Signaling Technology). Protein bands were detected after incubation with ECL solution (Thermo Fisher Scientific, Inc.) for $2 \mathrm{~min}$ in the dark. GAPDH served as a loading control and the densitometry of band intensities were detected by Amersham Imager 600 (GE Healthcare).

Cell cycle distribution analysis. After the cells were washed twice and collected in $100 \mu \mathrm{l}$ prechilled PBS, they were fixed with $95 \%$ ethanol overnight at $4^{\circ} \mathrm{C}$. The next day, the cells were collected and washed once with prechilled PBS before they were treated with FxCycle PI/RNase Staining Solution (Invitrogen; Thermo Fisher Scientific, Inc.). The cell cycle distribution was determined by a Flow cytometer (BD Biosciences) and analyzed by ModFit LT 3.2 (Verity Software House).

Alkaline comet assay. The cells were washed twice with prechilled PBS and diluted to $1.5 \times 10^{5} / \mathrm{ml}$. The comet assay was performed using CometAssay HT kits (Trevigen). Aliquots of $50 \mu \mathrm{l}$ of cells $(7,500$ cells total) were mixed with $500 \mu \mathrm{l}$ of LM-Agarose and rapidly pipetted over the entire sample area of comet slides, which were then placed at $4^{\circ} \mathrm{C}$ for $30-45 \mathrm{~min}$ in the dark. After the agarose solidified, the slides were immersed in ice-cold lysis solution for $2 \mathrm{~h}$ at $4^{\circ} \mathrm{C}$ in the dark. Excess buffer was drained after lysis, and the slides were immersed in freshly prepared alkaline unwinding solution for $30 \mathrm{~min}$ at room temperature in the dark. The slides were placed in an electrophoresis slide tray, covered with slide tray overlay and electrophoresed at $21 \mathrm{~V}$ for $25 \mathrm{~min}$. After electrophoresis, the slides were washed twice with deionized water, immersed in $70 \%$ ethanol for $10 \mathrm{~min}$ in the dark, and placed in a drying oven for $30 \mathrm{~min}$ to remove excess buffer. SYBR Gold was pipetted onto each sample, and the slides were incubated for
$30 \mathrm{~min}$ at room temperature in the dark. Images were collected by an Olympus microscope and charge-coupled device (CCD) camera at a final magnification of x200. The tail moment was analyzed using the Comet Assay Software Project 4 (CASP 4; Perspective Instruments, Ltd.) as an indicator of DNA damage.

Colony formation assay. K562 cells were seeded evenly at 200 cells/well in 24-well plates. A two-layer soft agarose assay was performed with $0.6 \%$ agarose as the bottom layer and $0.3 \%$ agarose as the top layer. After the cells were seeded, $100 \mu \mathrm{l}$ of culture medium was added to each well every 2 days. After 10 days, colony number and size were analyzed, and representative images were captured by microscope (Nikon) and charge-coupled device (CCD) camera.

CCK- 8 assay. Cells were seeded in 96-well plates and treated as indicated. Then, $10 \mu \mathrm{l}$ of CCK-8 reagent (Dojindo) was added to each well, and the plates were incubated for $2 \mathrm{~h}$ at $37^{\circ} \mathrm{C}$ in the dark. The absorbance at $450 \mathrm{~nm}$ was detected by a SpectraMax microplate reader (Molecular Devices). The combination index (CI) between CCT245737 and VP16 was calculated through Chou-Talalay method by CompuSyn software (CompuSyn Inc., USA).

Statistical analysis. All statistical analyses were performed using GraphPad Prism 6.0 (GraphPad Software, Inc.). Data are presented as the mean \pm standard deviation. All quantitative experiments were conducted with a minimum of three independent experiments. An unpaired two-tailed t test or one-way ANOVA followed by Turkey, Dunnett-T3 or Dunnett post-doc test was used as appropriate to determine statistical significance $\left({ }^{*} \mathrm{P}<0.05,{ }^{* *} \mathrm{P}<0.01\right.$, and $\left.{ }^{* * *} \mathrm{P}<0.001\right)$. Statistical analyses were performed with IBM SPSS Statistics 19.0 (SPSS Inc., USA) and GraphPad Prism 7 (GraphPad Software, USA).

\section{Results}

CHK1 displays higher expression in K562 cells. The KCL22 and K562 cell lines are the two most common CML cell lines used in experiments. To determine CHK1 mRNA levels in these two cell lines, we performed qPCR with CAM-191 cells as a control as they have the lowest CHK1 mRNA expression of the three cell lines tested (the dotted line). As shown in Fig. 1A, CHK1 mRNA expression was significantly higher in K562 cells than in KCL22 cells. Consistently, western blot analysis demonstrated that $\mathrm{CHK} 1$ protein expression was also significantly higher in K562 cells than in KCL22 cells (Fig. 1B and C). Thus, K562 cells were selected for the subsequent experiments.

shRNA-mediated CHK1 knockdown efficiently suppresses cell proliferation. To determine the role of CHK1 in K562 cell proliferation, we transduced K562 cells with lentiviral vectors containing shRNA specifically targeting CHK1 (shCHK1-KD), a negative control sequence (shRNA-NC) or an empty pLVX-shRNA vector (shRNA-Vector). shCHK1-KD significantly reduced $\mathrm{CHK} 1$ expression at both the mRNA and protein levels (Fig. 2A and B). Compared with shRNA-NC and shRNA-Vector, shCHK1-KD significantly suppressed K562 cell proliferation (Fig. 2C). Thus, CHK1 is a potential target for the treatment of CML. 
A

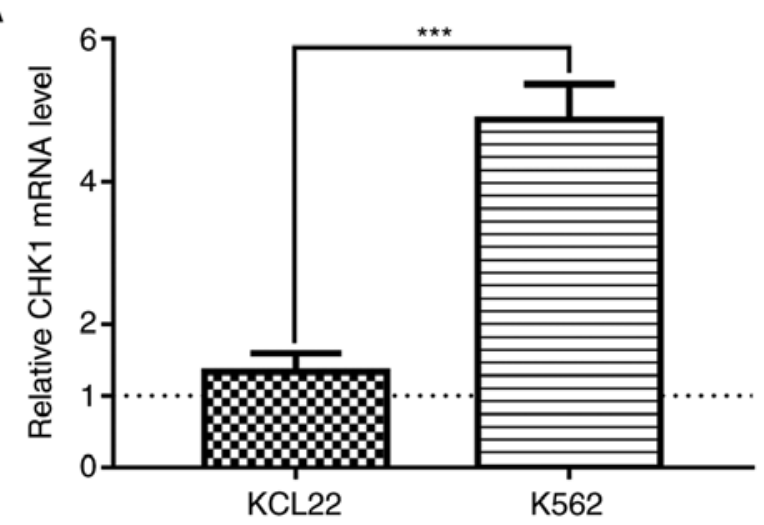

B

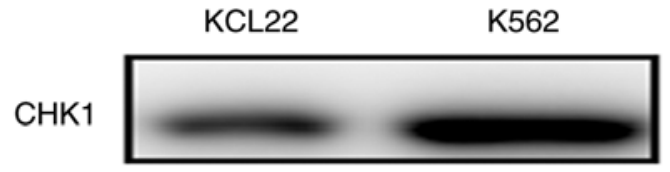

GAPDH

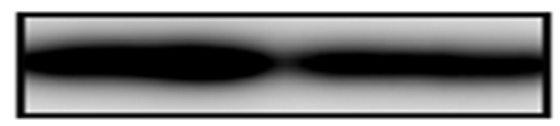

C

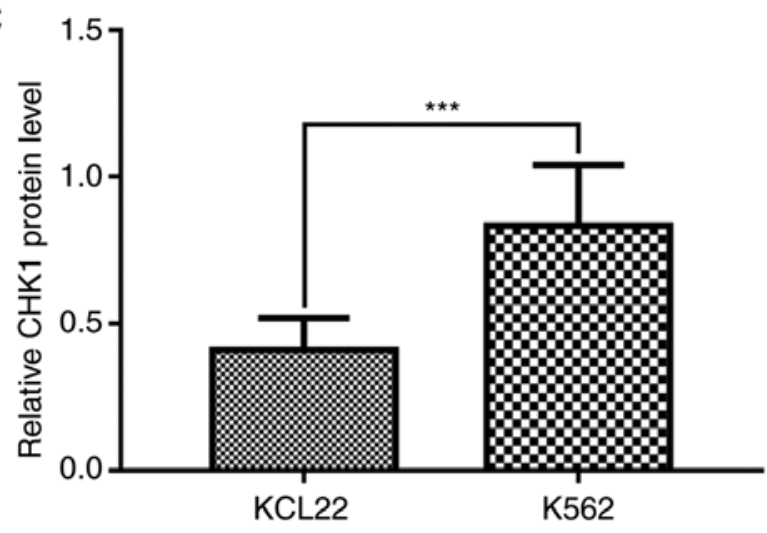

Figure 1. CHK1 expression is higher in K562 cells. (A) CHK1 mRNA levels relative to $\beta$-actin (loading control) levels in KCL22 and K562 cells were detected by qPCR, CAM-191 cells were used as the control group which is shown as a dotted line. (B) Western blot analysis of CHK1 protein levels in KCL22 and K562 cells. (C) Relative quantification of CHK1 expression normalized to GAPDH expression based on densitometric analysis of the band intensities shown in $\mathrm{B}\left({ }^{* * *} \mathrm{P}<0.001\right)$ comparison between KCL22 and K562 cells. CHK1, checkpoint kinase 1.

CHK1 knockdown enhances the cytotoxicity of VP16 in K562 cells. To investigate whether silencing CHK1 increases the cytotoxicity of VP16 in K562 cells, K562 cells were transduced with shCHK1-KD, shRNA-NC or shRNA-Vector and then treated with $5 \mu \mathrm{M}$ VP16; we then analyzed DSBs, apoptosis and colony formation. First, we investigated the level of phosphorylated $\mathrm{H} 2 \mathrm{AX}(\gamma \mathrm{H} 2 \mathrm{AX})$, a protein marker of DSBs. The results demonstrated that CHK1 knockdown increased $\gamma \mathrm{H} 2 \mathrm{AX}$ levels compared to the two controls, especially when combined with VP16 treatment (Fig. 3A). Subsequently, we performed comet assays and analyzed different parameters using CASP. The Olive Tail Moment (OTM) was used to describe the level of DNA damage in cells. Similar to the change in $\gamma \mathrm{H} 2 \mathrm{AX}$ accumulation, the comet assay showed that CHK1 knockdown slightly, but not obviously, increased
A

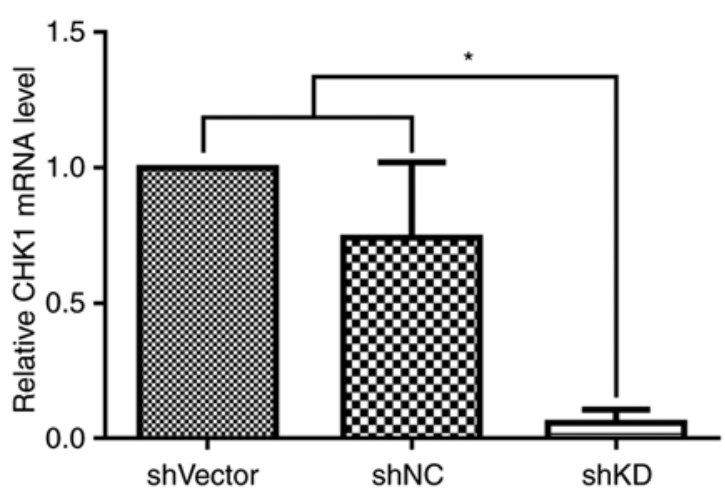

B

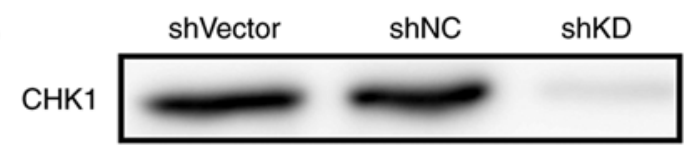

GAPDH

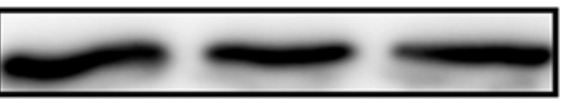

C

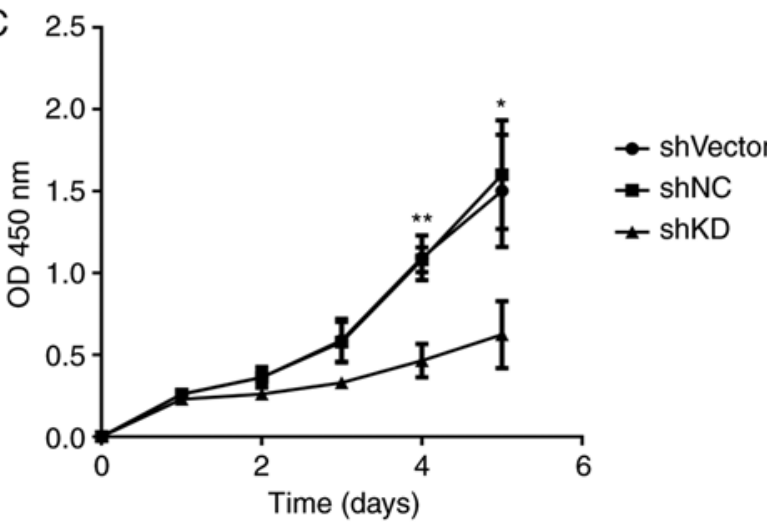

Figure 2. shRNA-mediated CHK1 knockdown efficiently suppresses cell proliferation. (A) CHK1 mRNA levels relative to $\beta$-actin (loading control) levels in K562 cells infected with three different lentiviruses (shVector, shNC and shKD) as detected by qPCR. (B) Western blotting was performed to confirm that shCHK1 (shKD) efficiently knocked down CHK1 protein expression in K562 cells. (C) Cell viability on different days was examined by CCK-8 assays after Chk1 knockdown (shKD). The OD450 $\mathrm{nm}$ values were measured and plotted with respect to time $\left({ }^{*} \mathrm{P}<0.05,{ }^{* *} \mathrm{P}<0.01\right)$. CHK1, checkpoint kinase 1. shKD, Chk1-knockdown cells.

OTM values under both normal growth and DMSO treatment conditions. However, after VP16 treatment, the CHK1 knockdown group exhibited a significant increase in DNA damage (Fig. 3B and C). These results revealed that CHK1 knockdown can increase VP16-induced DNA damage in K562 cells. Next, as shown in Fig. 3D, CHK1 knockdown did not significantly increase the expression of cleaved poly(ADP-ribose) polymerase (c-PARP), an apoptosis marker, under either normal growth or DMSO treatment conditions. However, after incubation with VP16, cells with CHK1 knockdown showed distinctly increased levels of c-PARP, which indicates elevated levels of apoptosis. Furthermore, we performed colony formation assays to determine the role of CHK1 in cell proliferation. Silencing of CHK1 was associated with a significant decrease in colony formation ability under all conditions, regardless of 
A

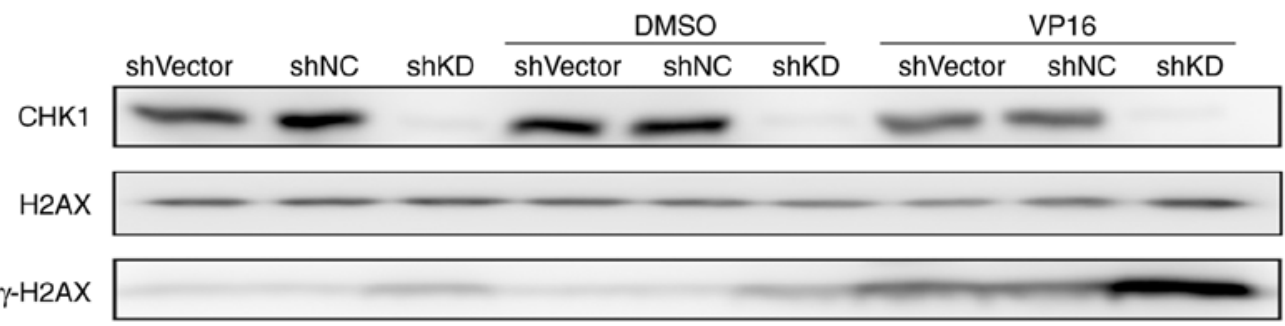

GAPDH

B

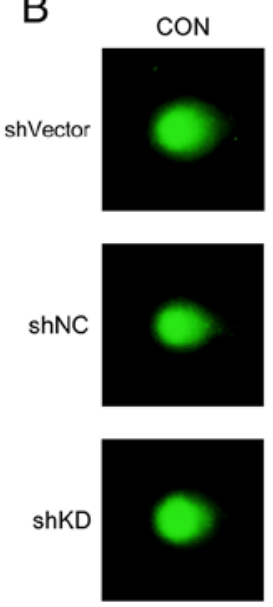

DMSO
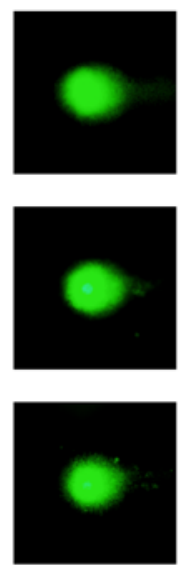

C
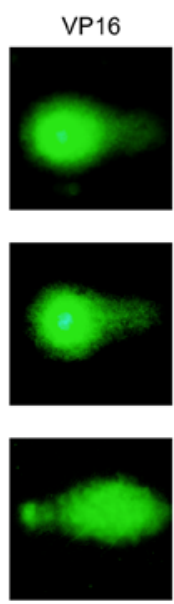

D

\begin{tabular}{|c|c|c|c|c|c|c|c|c|}
\hline \multirow[b]{2}{*}{ shVector } & \multirow[b]{2}{*}{ shNC } & \multirow[b]{2}{*}{ shKD } & \multicolumn{3}{|c|}{ DMSO } & \multicolumn{3}{|c|}{ VP16 } \\
\hline & & & shVector & shNC & shKD & shVector & shNC & shKD \\
\hline
\end{tabular}

c-PARP

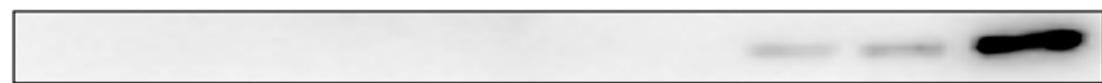

GAPDH

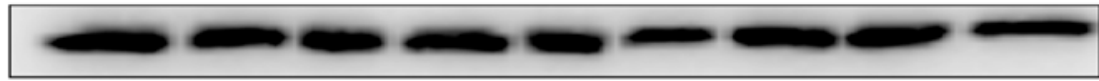

E

CON

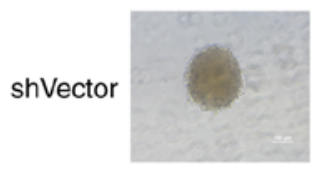

$\operatorname{shNC}(2)$

shKD

\section{DMSO}
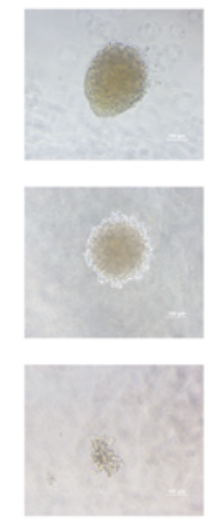

VP16

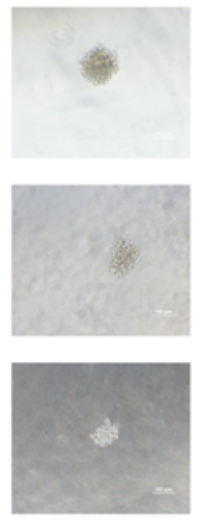

$\mathrm{F}$

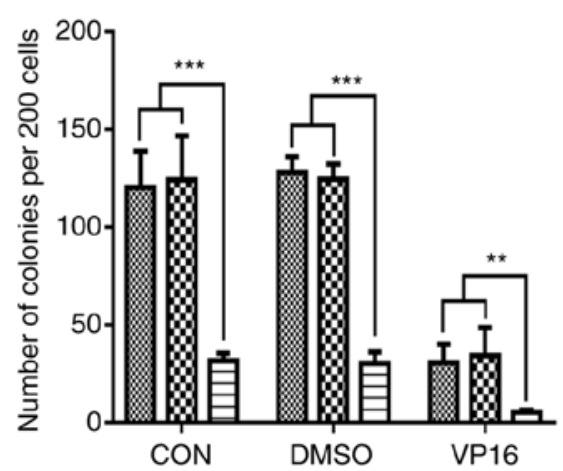

shVector

$\mathbb{B}$ shNC

是K $\mathrm{sh}$

Figure 3. CHK1 knockdown enhances the cytotoxicity of VP16 in K562 cells. (A) Western blot analysis of the expression of the DSB marker $\gamma \mathrm{H} 2 \mathrm{AX}$ and total H2AX. (B) An alkaline comet assay was performed using cells transduced with shVector, shNC, or shCHK1 (shKD) under normal conditions (CON) or after treatment with DMSO or VP16 to assess the level of DNA damage. CHK1 silencing by shRNA enhanced the induction of DNA damage by VP16. (C) Graphical presentation of the tail moment in the alkaline comet assay described in $\mathrm{B}\left({ }^{* * *} \mathrm{P}<0.001\right)$. (D) Western blot analysis of cleaved PARP (c-PARP), an apoptosis marker. shRNA-mediated CHK1 inhibition significantly increased VP16-induced apoptosis. (E) A colony formation assay was performed in K562 cells infected with three different lentiviruses under normal conditions (CON) and after treatment with DMSO or VP16. (F) Graphical presentation of the number of colonies described in $\mathrm{E}\left({ }^{* *} \mathrm{P}<0.01,{ }^{* * *} \mathrm{P}<0.001\right)$. NS, not significant; CHK1, checkpoint kinase 1; DSB, double-strand break; VP16, etoposide. 
A

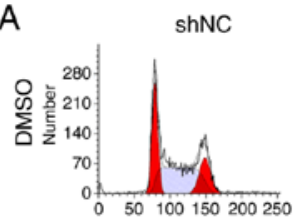

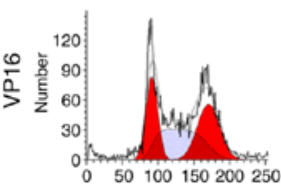
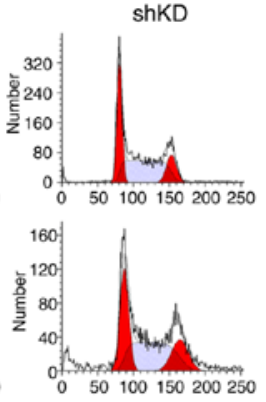

C

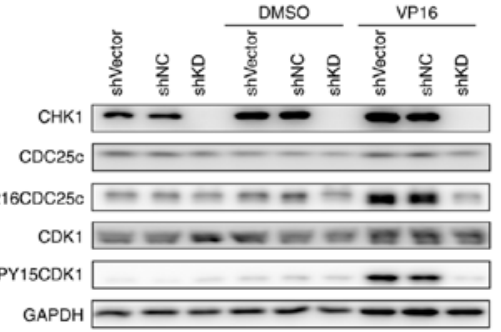

$\mathrm{E}$

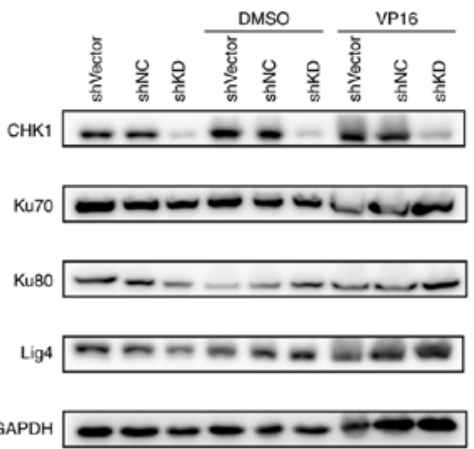

B
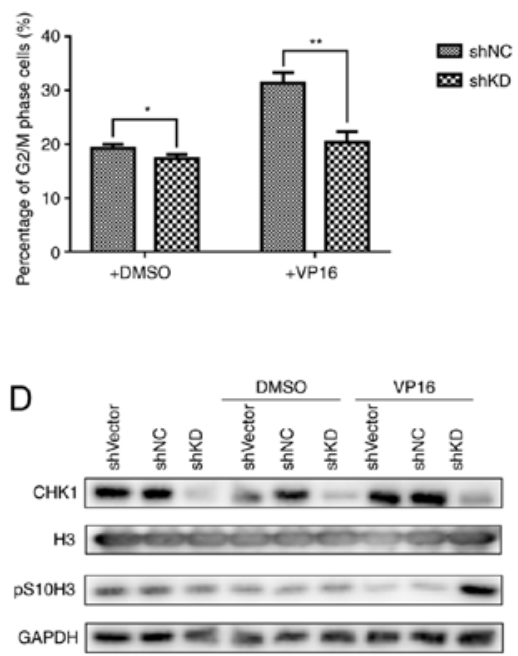

G

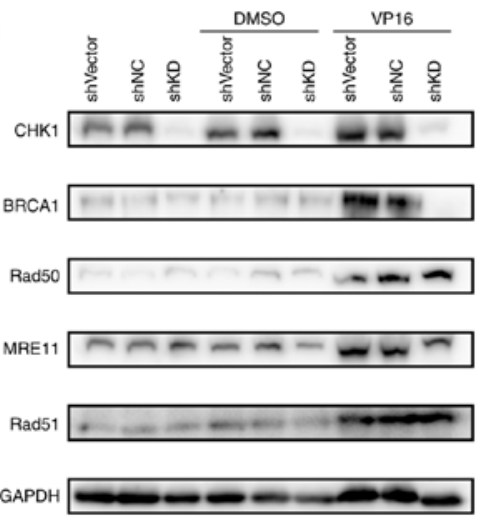

F
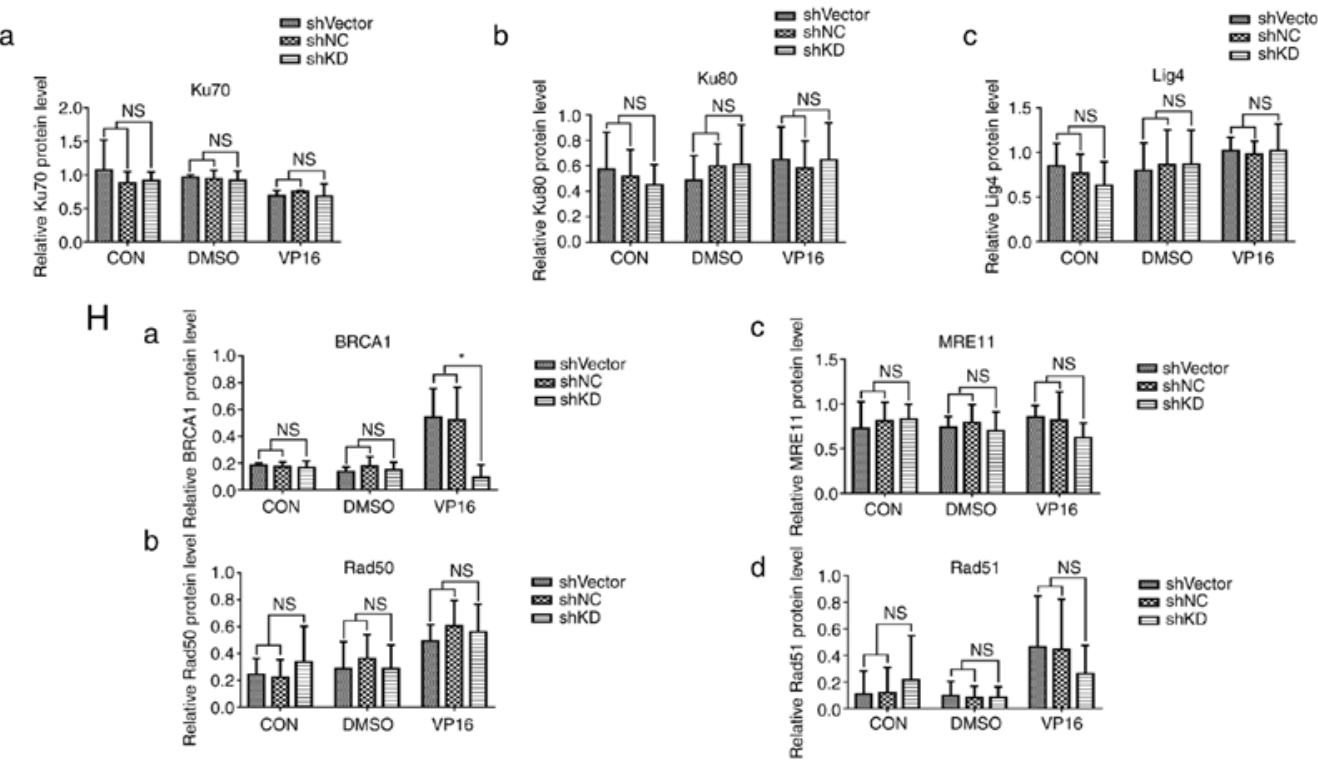

Figure 4. shRNA-mediated CHK1 knockdown reduces $\mathrm{G}_{2} / \mathrm{M}$ arrest and HR repair in K562 cells. (A) The cell cycle distribution of K562 cells transduced with

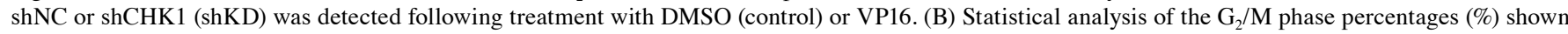
in $\mathrm{A}\left({ }^{*} \mathrm{P}<0.05,{ }^{* *} \mathrm{P}<0.01\right)$. (C) Western blot analysis of the $\mathrm{G}_{2} / \mathrm{M}$ arrest markers pS216CDC25c, pY15CDK1 and total CDC25c and CDK1 in K562 cells infected with lentivirus carrying shVector, shNC or shCHK1 (shKD) under normal conditions and in the presence of DMSO or VP16. (D) Western blot analysis of the mitotic marker pS10-H3 and total H3 in K562 cells infected with lentivirus carrying shVector, shNC or shCHK1 (shKD) under normal conditions and in the presence of DMSO or VP16. (E) Western blot analysis of the NHEJ pathway-related proteins Ku70, Ku80 and ligase 4 (Lig4) in shVector-, shNC-, or shCHK1-transduced (shKD) K562 cells under normal conditions and after treatment with DMSO or VP16. (F) Relative quantification of Ku70 (a), Ku80 (b) and Lig4 (c) expression shown in E by densitometric analysis; target band intensity was normalized to that of GAPDH. (G) Western blot analysis of the HR pathway-related proteins BRCA1, Rad50, MRE11 and Rad51 in shVector-, shNC-, or shCHK1-transduced (shKD) K562 cells under normal conditions and after treatment with DMSO or VP16. (H) Relative quantification of BRCA1 (a), Rad50 (b), MRE11 (c) and Rad51 (d) expression shown in G by densitometric analysis; band intensity was normalized to that of GAPDH ( $\left.{ }^{*} \mathrm{P}<0.05\right)$. NS, not significant. CHK1, checkpoint kinase 1; HR, homologous recombination; VP16, etoposide. 
A

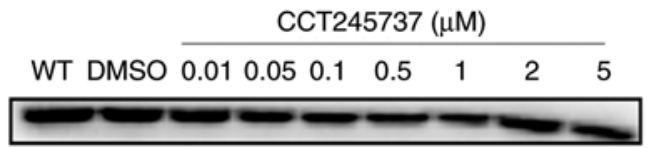

GAPDH

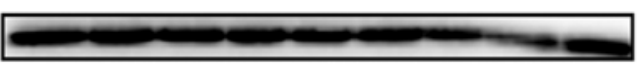

B

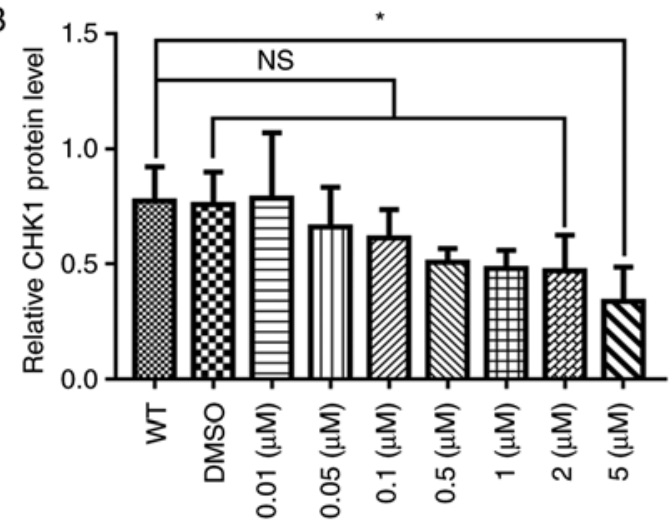

C
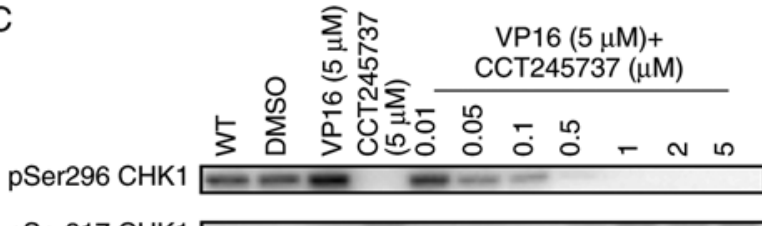

pSer317 CHK1 — = - = = =

pSer345 CHK1
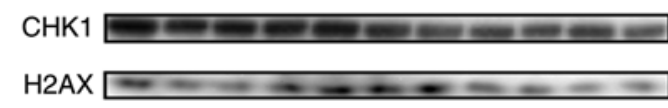

$\gamma \mathrm{H} 2 \mathrm{AX}$

GAPDH

Figure 5. Characterization of the effects of CCT245737 on the VP16-induced changes in the levels of CHK1 and DNA damage biomarkers in K562 cells. (A) Western blot analysis of total CHK1 expression in K562 cells after treatment with increasing concentrations of CCT245737 for $24 \mathrm{~h}$. (B) Relative quantification of total CHK1 expression shown in A by densitometric analysis; band intensity was normalized to that of GAPDH ("P $<0.05)$. (C) Western blot analysis of K562 cells treated with VP16 $(5 \mu \mathrm{M})$, CCT245737 $(5 \mu \mathrm{M})$ or the combination of VP16 and increasing concentrations of CCT245737. CHK1 autophosphorylation at Ser296 and phosphorylation at Ser317 and Ser345 were used as biomarkers of CHK1 activity, and $\gamma \mathrm{H} 2 \mathrm{AX}$ was used as a biomarker of DNA damage. NS, not significant. CHK1, checkpoint kinase 1; VP16, etoposide.

the presence of VP16 (Fig. 3E and F). These results suggest that downregulation of CHK1 enhances the cytotoxic effect of VP16 on K562 cells.

shRNA-mediated CHK1 knockdown reduces $G_{2} / M$ arrest and $H R$ repair in K562 cells. Accurate and timely DNA damage repair is vital for cells to survive chemotherapy-induced DNA damage. As we showed that CHK1 knockdown sensitized K562 cells to VP16, we aimed to ascertain whether CHK1 knockdown impairs the DNA damage repair pathway in K562 cells. First, we analyzed the cell cycle distribution in the shRNA-NC and shCHK1-KD groups and found that CHK1 knockdown eliminated $\mathrm{G}_{2} / \mathrm{M}$ arrest under both conditions, especially following
VP16 treatment (Fig. 4A and B). Consistent with the cell cycle distribution results, VP16 treatment increased the levels of the $\mathrm{G}_{2} / \mathrm{M}$ arrest markers pS216 CDC25c and pY15 CDK1, and shRNA-mediated CHK1 knockdown suppressed the induction of pS216 CDC25c and pY15 CDK1 by VP16 (Fig. 4C). Moreover, CHK1 knockdown reversed the VP16-induced decrease in the levels of the mitotic marker pS10 H3 (Fig. 4D). These results indicate that shRNA-mediated CHK1 knockdown mitigates chemotherapy-induced $\mathrm{G}_{2} / \mathrm{M}$ arrest and forces cells to enter mitosis with unrepaired DNA damage. HR and non-homologous end joining (NHEJ) are two major pathways for repairing damaged DNA. Although a previous study showed that $\mathrm{CHK} 1$ is required for the HR repair pathway (17), the detailed mechanism is still unclear. Here, we examined the expression of proteins related to NHEJ and HR to determine which pathway is affected by CHK1 silencing. CHK1 knockdown had no impact on the expression of the NHEJ-related proteins Ku70/80 and ligase 4 (Lig4) (Fig. 4E and F). In contrast, the HR pathway was impaired based on the suppression of VP16-induced BRCA1 elevation, which represents the location capacity of Rad51 to DNA damage loci. However, shRNA-mediated CHK1 ablation had no direct influence on the VP16-mediated induction of the HR-related proteins MRE11, Rad50 and Rad51 (Fig. 4G and H). Taken together, the results showed that CHK1 knockdown abolishes $\mathrm{G}_{2} / \mathrm{M}$ arrest and reduces the efficiency of HR repair; thus, knockdown cells are forced to enter mitosis with unrepaired DNA damage.

CCT245737 efficiently inhibits CHK1 phosphorylation and promotes the accumulation of DNA damage. CCT245737 is a potent CHK1 inhibitor with good oral bioavailability. As shown in a previous study $(26,27)$, CCT245737 is a selective CHK1 inhibitor with a half maximal inhibitory concentration $\left(\mathrm{IC}_{50}\right)$ value of $1.4 \pm 0.3 \mathrm{nM}$ and $>1,000$-fold selectivity over CHK2 and CDK1.

To confirm the ability of CCT245737 in inhibiting CHK1, we first analyzed total CHK1 protein expression in cells treated with different concentrations of CCT245737. As shown in Fig. 5A and B, increasing concentrations of CCT245737 did not significantly decrease total CHK1 protein levels, and CHK1 was inhibited at only a high concentration of CCT245737 $(5 \mu \mathrm{M})$. Then, we treated K562 cells with VP16 and increasing concentrations of CCT245737 alone or in combination to investigate whether CCT245737 influences phosphorylated CHK1 levels. VP16 obviously induced CHK1 autophosphorylation at Ser296 and phosphorylation at Ser317 and Ser345 (Fig. 5C). In the presence of CCT245737, VP16-induced Ser296 autophosphorylation was dramatically suppressed at $0.05 \mu \mathrm{M}$ and completely eliminated at $0.5 \mu \mathrm{M}$. In contrast, the influence of CCT245737 on other CHK1 phosphorylation sites (Ser317 and Ser345) and total CHK1 levels was minimal. Meanwhile, the inhibition of CHK1 autophosphorylation at Ser296 coincided with the accumulation of $\gamma \mathrm{H} 2 \mathrm{AX}$. Therefore, these results suggest that CCT245737 can efficiently inhibit the activation of CHK1 by suppressing VP16-induced CHK1 autophosphorylation at Ser296 and promoting the accumulation of DNA damage in VP16-treated K562 cells.

CCT245737 sensitizes K562 cells to VP16. To determine the cytotoxicity of CCT245737, K562 cells were treated 

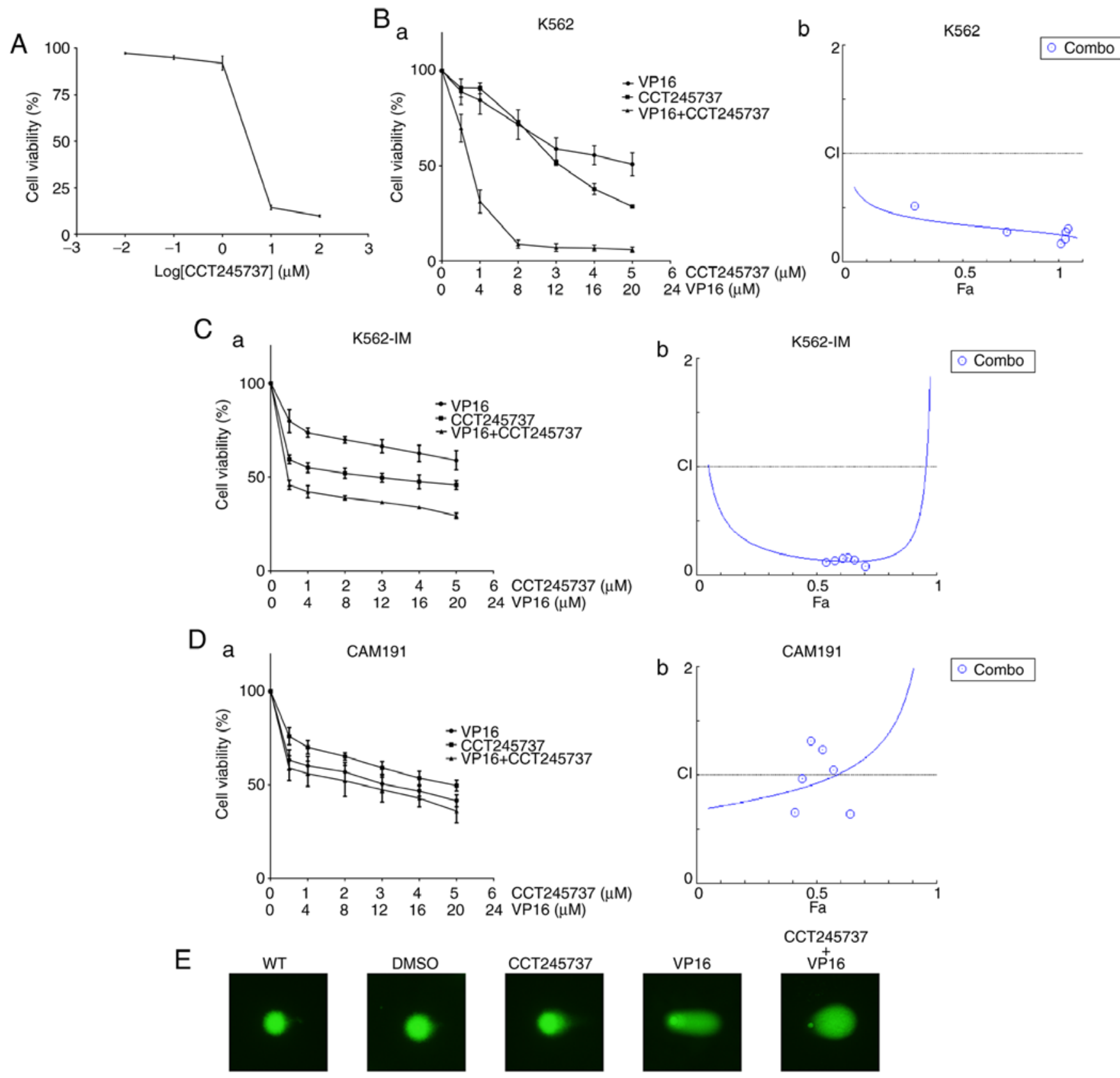

$\mathrm{E}$
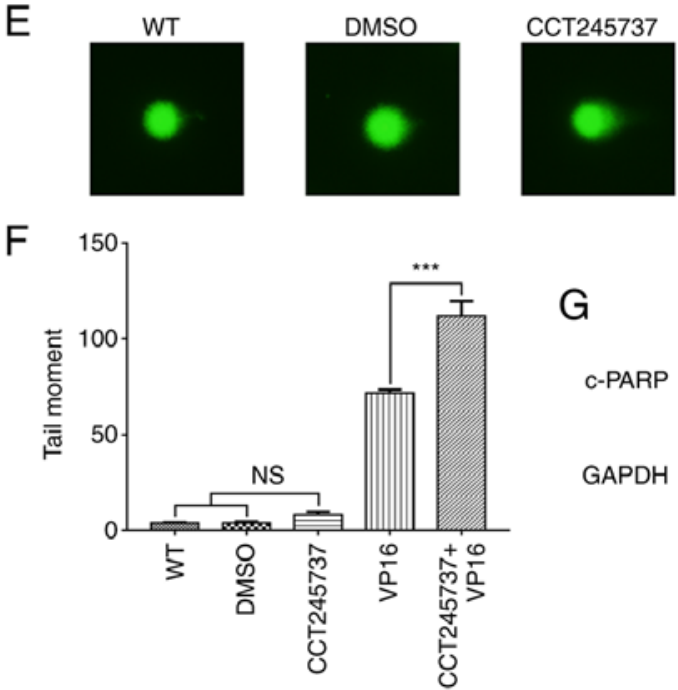

G

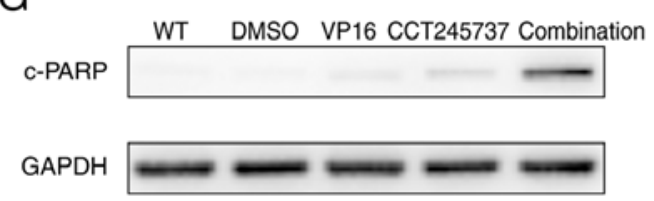

Figure 6. CCT245737 sensitizes K562 cells to VP16. (A) Graphical presentation of the \% viability of K562 cells after treatment with increasing concentrations of CCT245737 for 24 h. (B) Graphical presentation of (a) the \% viability and (b) the Fa-CI after Chou-Talalay analysis of K562 cells after treatment with the indicated drugs for $24 \mathrm{~h}$. (C) Graphical presentation of (a) the \% viability and (b) the Fa-CI after Chou-Talalay analysis of imatinib-treated K562 (K562-IM) cells after treatment with the indicated drugs for $24 \mathrm{~h}$. (D) Graphical presentation of (a) the \% viability and (b) the Fa-CI after Chou-Talalay analysis of CAM191 cells after treatment with the indicated drugs for $24 \mathrm{~h}$. (E) An alkaline comet assay was performed to assess the extent of DNA damage in K562 cells treated with CCT245737 $(500 \mathrm{nM})$ and/or VP16 $(5 \mu \mathrm{M})$ for $24 \mathrm{~h}$. (F) Graphical presentation of the tail moment in the alkaline comet assay described in $\mathrm{E}\left({ }^{* * *} \mathrm{P}<0.001\right)$. (G) Western blot analysis of the apoptotic marker cleaved PARP in K562 cells treated with CCT245737 (500 nM) and/or VP16 (5 $\left.\mu \mathrm{M}\right)$ for $24 \mathrm{~h}$. The combination of VP16 with CCT245737 increased the levels of c-PARP. NS, not significant; VP16, etoposide; c-PARP, cleaved poly(ADP-ribose) polymerase. 
A
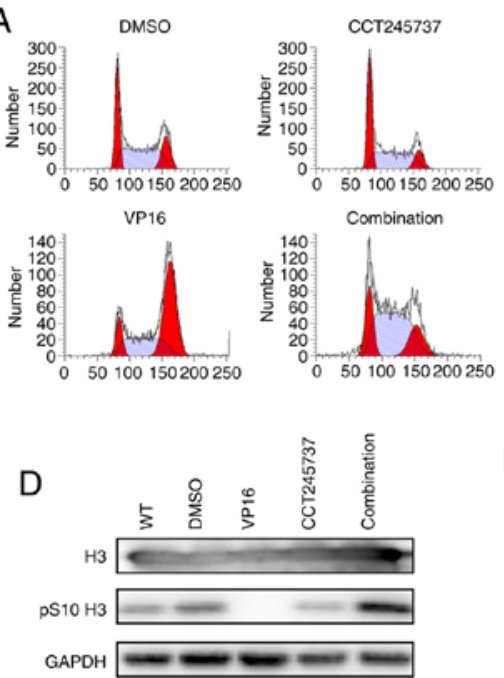

$\mathrm{F}$

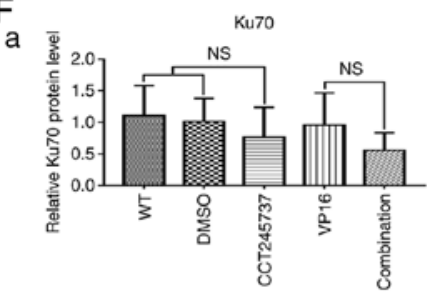

b

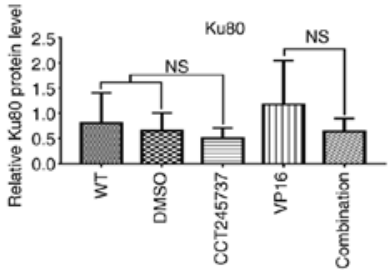

C
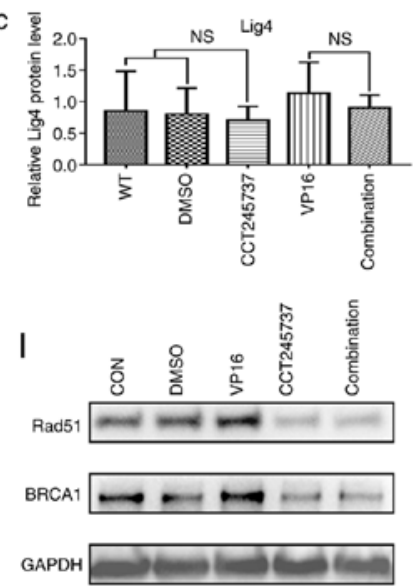

B

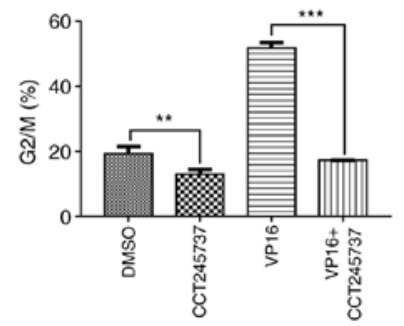

E
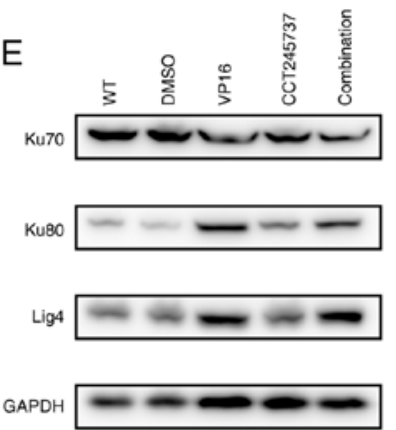
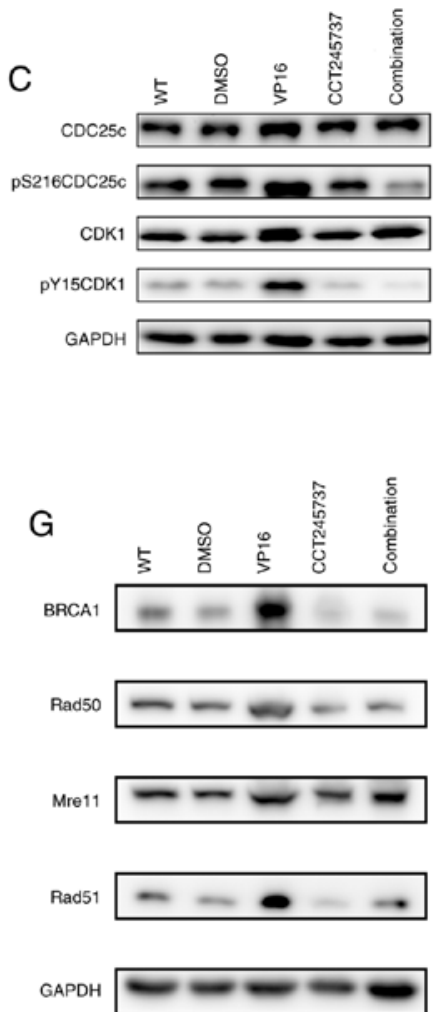

C
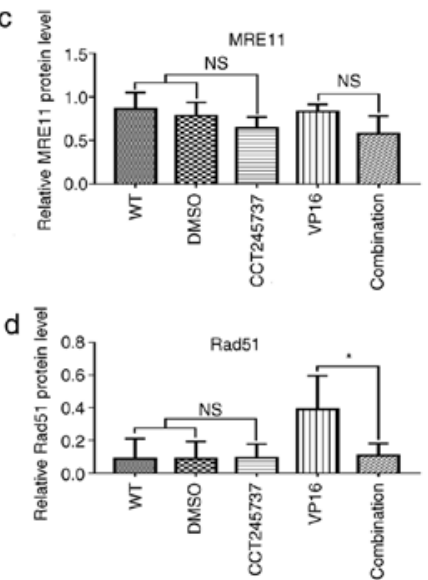
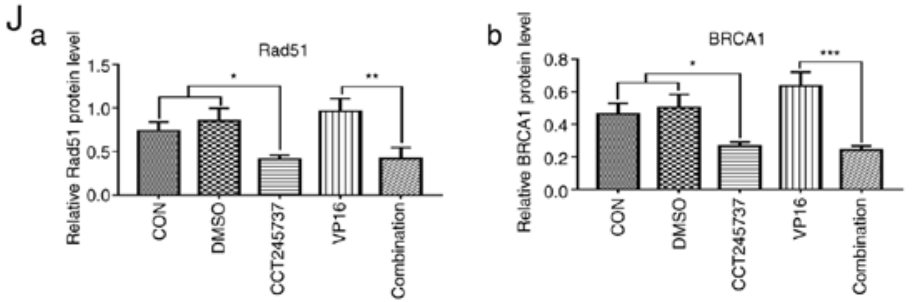

Figure 7. CCT245737 abrogates $\mathrm{G}_{2} / \mathrm{M}$ arrest and reduces HR repair capacity in K562 cells. (A) Effects of CCT245737 (500 nM, $24 \mathrm{~h}$ ) on cell cycle arrest in K562 cells treated with acute VP16 $(5 \mu \mathrm{M}, 1.5 \mathrm{~h})$. (B) Statistical analysis of the $\mathrm{G}_{2} / \mathrm{M}$ phase percentage $(\%)$ described in $\mathrm{A}\left({ }^{* * *} \mathrm{P}<0.01,{ }^{* * * *} \mathrm{P}<0.001\right)$. (C) Western blot analysis of the $\mathrm{G}_{2} / \mathrm{M}$ arrest markers pS216CDC25c, pY15CDK1 and total CDC25c and CDK1 in K562 cells treated with CCT245737 (500 nM) and/or VP16 $(5 \mu \mathrm{M})$ for $24 \mathrm{~h}$. (D) Western blot analysis of the mitosis marker pS10-H3 and total H3 in K562 cells treated with CCT245737 (500 nM) and/or VP16 $(5 \mu \mathrm{M})$ for $24 \mathrm{~h}$. (E) Western blot analysis of the NHEJ pathway-related proteins Ku70, Ku80 and ligase 4 (Lig4) in K562 cells treated with CCT245737 $(500 \mathrm{nM})$ and/or VP16 $(5 \mu \mathrm{M})$ for $24 \mathrm{~h}$. (F) Relative quantification of Ku70 (a), Ku80 (b) and Lig4 (c) expression shown in E by densitometric analysis of band intensities normalized to those of GAPDH. (G) Western blot analysis of the HR pathway-related proteins BRCA1, Rad50, MRE11 and Rad51 in K562 cells treated with CCT245737 (500 nM) and/or VP16 (5 $\mu \mathrm{M})$ for $24 \mathrm{~h}$. (H) Relative quantification of BRCA1 (a), Rad50 (b), MRE11 (c) and Rad51 (d) expression shown in $\mathrm{G}$ by densitometric analysis of band intensities normalized to those of GAPDH ("P<0.05). (I) Western blot analysis of BRCA1 and Rad51 protein expression in imatinib-treated K562 cells after exposure to CCT245737 (500 nM) and/or VP16 (5 $\mu \mathrm{M})$ for $24 \mathrm{~h}$. (J) Relative quantification of Rad51 (a) and BRCA1 (b) expression shown in I by densitometric analysis of band intensities normalized to those of GAPDH ("P<0.05, $\left.{ }^{* *} \mathrm{P}<0.01,{ }^{* * *} \mathrm{P}<0.001\right)$. NS, not significant; HR, homologous recombination; CHK1, checkpoint kinase 1; VP16, etoposide. 


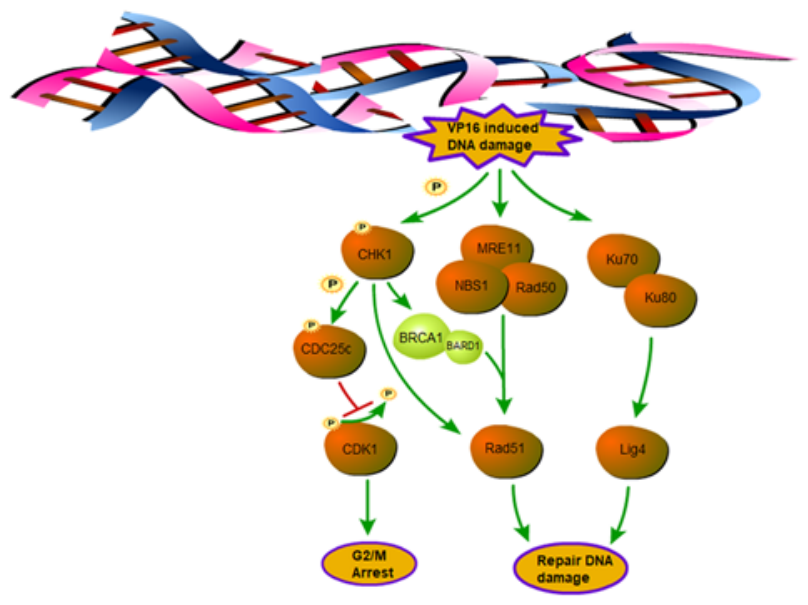

Figure 8. Schematic model of the CHK1-mediated regulation of the $\mathrm{G}_{2} / \mathrm{M}$ cell cycle checkpoint and HR repair pathway. BRCA1, breast cancer gene susceptibility 1; BARD1, BRCA1-associated ring domain protein 1; CDC25c, cell division cyclin 25 homolog C; CDK1, cyclin dependent kinase 1; CHK1, checkpoint kinase 1; Lig4, ligase 4.

with increasing concentrations of CCT245737 for $24 \mathrm{~h}$, and cell viability was measured by CCK- 8 assays. Our results showed that K562 cell viability was significantly reduced by CCT245737 in a dose-dependent manner (Fig. 6A). Furthermore, we demonstrated that CCT245737 and VP16 had a synergistic effect on the viability of both K562 and imatinib-treated K562 cells (K562-IM), with a CI of 0.35 and 0.15 at a $50 \%$ effective dose $\left(\mathrm{ED}_{50}\right)$ as calculated by the Chou-Talalay analysis (Fig. 6B and C) (31). To clarify that this drug combination is specific for cancer cells, we performed the same experiment with a normal human lymphocyte cell line, CAM191. CCT245737 showed weak synergism with VP16 in CAM191 cells compared with vehicle- and imatinib-treated $\mathrm{K} 562$ cells, with a $\mathrm{CI}$ of 0.93 at the $\mathrm{ED}_{50}$ (Fig. 6D). Moreover, consistent with the $\gamma \mathrm{H} 2 \mathrm{AX}$ accumulation shown in Fig. 4C, comet assays demonstrated that CCT245737 obviously increased the level of VP16-induced DNA damage (Fig. 6E and F). Furthermore, the increased c-PARP levels in the combination treatment group suggested the upregulation of apoptosis (Fig. 6G). These data revealed that CCT245737 may have a considerable and specific synergistic anticancer effect with VP16 in K562 cells treated with or without imatinib.

CCT245737 abrogates $G_{2} / M$ arrest and reduces $H R$ repair capacity in $\mathrm{K} 562$ cells. To elucidate the mechanism of the anticancer synergy between CCT245737 and VP16 in leukemia cells, we first analyzed the cell cycle distribution. CCT245737 abolished $\mathrm{G}_{2} / \mathrm{M}$ arrest in $\mathrm{K} 562$ cells under normal conditions and after acute VP16 exposure $(5 \mu \mathrm{M}$ for $1.5 \mathrm{~h}$ ) (Fig. 7A and B). The western blot results showed that CCT245737 efficiently reduced the VP16-induced increases in the $\mathrm{G}_{2} / \mathrm{M}$ arrest markers pS216 CDC25c and pY15 CDK1 (Fig. 7C). Moreover, the VP16-induced decrease in the mitotic marker pS10 H3 was rescued by CCT245737 (Fig. 7D). Next, the expression of critical proteins related to the NHEJ and HR pathways was investigated to elucidate the potential mechanism by which CCT245737 enhances chemosensitivity. Consistent with the results of shRNA-induced CHK1 silencing, CCT245737 had no obvious impact on NHEJ-related protein expression (Fig. 7E and F). However, in contrast to the results of the shCHK1-KD experiments, the induction of both Rad51 and BRCA1 by VP16 was markedly suppressed by CCT 245737 (Fig. 7G and H). This result indicates that CCT245737 does not only reduce the local availability of Rad51 but also directly suppresses Rad51 expression after DSBs. Due to the results above, BRCA1 and Rad51 were chosen to be the most important proteins involved in CHK1-related DNA damage repair pathway in K562 wild-type (K562-WT) cells. Thus, we prioritized the Rad51 and BRCA1 expression in imatinib-treated K562 (K562-IM) cells over other assays to certify that CHK1 inhibition by CCT245737 could also suppress HR efficiency in K562-IM cells through downregulating these two proteins expression and similar results were obtained in K562-IM cells. Compared with K562-WT cells, K562 cells pretreated with imatinib showed a nonsignificant VP16-induced increase in Rad51 and BRCA1 expression, perhaps because this pretreatment could induce abundant Rad51 and BRCA1 expression in these cells. CCT245737 also obviously inhibited Rad51 and BRCA1 expression in imatinib-treated K562 cells, indicating that CCT245737 can also effectively suppress HR efficiency in TKI-treated CML cells (Fig. 7I and J). In conclusion, these results suggest that CCT245737 can abolish the VP16-induced activation of the $\mathrm{G}_{2} / \mathrm{M}$ checkpoint, efficiently inhibit the HR repair pathway, and promote the progression of cells with unrepaired DNA damage into mitosis.

\section{Discussion}

Although the development of various chemotherapeutic drugs has greatly improved cancer therapy, there remain patients who are resistant to certain therapies. Due to the critical role of the DNA damage response (DDR) in chemotherapy resistance, blocking this pathway has emerged as a new approach to address this problem. Checkpoint kinase 1 (CHK1), a well-known cell cycle checkpoint protein, is associated with the DDR pathway, especially in p53-mutant cells, which account for a large subset of cancer cells $(6,32)$. CHK1 is involved in not only cell cycle regulation but also the homologous recombination (HR) pathway through direct or indirect regulation of Rad51 (Fig. 8). Thus, CHK1 is potentially an ideal candidate for the treatment of chemotherapy-resistant cancer.

CCT245737, a selective CHK1 inhibitor with greater than 1,000-fold selectivity over CHK2 and CDK1, has an oral bioavailability nearly $100 \%$ (26). The present study demonstrated that CCT245737 suppressed CHK1 activation by abolishing autophosphorylation at Ser296 but had a minimal impact on Ser317 and Ser345 phosphorylation or total CHK1 levels. As pSer317 CHK1 and pSer345 CHK1 levels are influenced by the levels of DNA damage and PP2A phosphatase activity, we utilized pSer296 CHK1 as a specific biomarker of CHK1 activity, consistent with previous studies $(19,33)$. Therefore, CCT 245737 could be considered a candidate CHK1 inhibitor to enhance the efficacy of chemotherapy.

In the present study, we first downregulated CHK1 expression in CML cells with shRNA and found that CHK1 silencing significantly increased DNA damage, promoted apoptosis, and reduced the proliferation and colony formation of cells. Subsequently, CCT245737 was shown to have a favorable and 
specific synergistic effect with VP16 in CML cells. To uncover the potential mechanism, we performed related experiments to investigate the influence of CHK1 inhibition on the cell cycle and the DNA damage repair pathway.

VP16, a DNA topoisomerase II (TOP2) inhibitor that can induce and stabilize DNA double-strand breaks (DSBs) by disrupting the TOP2 catalytic cycle at the DNA ligation step, is commonly included in anticancer therapy regimens (34). A previous study showed that VP16-mediated DSBs induce $\mathrm{G}_{2} / \mathrm{M}$ arrest by activating the $\mathrm{G}_{2} / \mathrm{M}$ cell cycle checkpoint to repair DNA damage (35). In our study, we revealed that both RNAi-mediated CHK1 silencing and CCT245737 efficiently abolished the VP16-induced activation of the $\mathrm{G}_{2} / \mathrm{M}$ cell cycle checkpoint, eliminating the time necessary for DNA repair by overruling the $\mathrm{G}_{2} / \mathrm{M}$ arrest. Hence, cells may enter mitosis with accumulated unrepaired damage.

Non-homologous end joining (NHEJ) and HR are the two major mechanisms for repairing DSBs (36). NHEJ can occur during almost the whole cell cycle, while HR only occurs in $S / G_{2}$ phase after DNA replication. In NHEJ repair, DSB ends are resected by various endonucleases or exonucleases to generate a microhomology sequence ( $<4$ nucleotides) (37). Then, Ku70 and Ku80 heterodimers (Ku70/80) recognize and bind DSBs to recruit nucleases, ligases, and polymerases, such as DNA ligase IV, to repair the breaks. We discovered that neither shRNA-mediated CHK1 knockdown nor CCT245737 significantly impacted the expression of Ku70/80 or DNA ligase IV, which indicated that the NHEJ repair pathway might not be influenced by CHK1 inhibition.

In contrast to NHEJ, which evokes error-prone repair, HR repair is a more precise method of repairing DNA damage. Once DSBs occur, the Mre11-Nbs1-Rad50 (MRN) complex converts the DSB ends into single-strand DNA (ssDNA). Subsequently, a key component of the HR pathway, $\operatorname{Rad} 51$, is recruited to replication protein A (RPA)-coated single-strand breaks (SSBs) and induces D-loop formation to repair the breaks. BRCA1 is involved in various biological processes, including mRNA splicing (38), DNA damage signaling (39) and HR repair $(40,41)$. BRCA1 can form a stable complex with BRCA1-associated ring domain protein 1 (BARD1) (42), and one study showed that it coimmunoprecipitates with Rad51 (43). A more recent report indicated that the BRCA1-BARD1 complex can directly interact with human Rad51, enhance the affinity of Rad51 for DNA damage loci and facilitate D-loop formation (44). In our study, shRNA-mediated Chk1 silencing markedly inhibited BRCA1 expression but not MRE11, Rad50 and Rad51 expression after VP16 treatment. Thus, shRNA-mediated CHK1 downregulation may suppress HR repair efficiency by reducing BRCA1-induced Rad51 localization and D-loop formation rather than by directly repressing Rad51 expression. However, in contrast to shRNA-mediated CHK1 inhibition, which only suppressed BRCA1 induction by VP16,CCT245737 considerably decreased the VP16-induced increases in both Rad51 and BRCA1 levels. A similar result was also found in imatinib-treated K562 cells. These results demonstrate that CCT245737 might block the HR pathway by both directly suppressing Rad51 expression and indirectly inhibiting the BRCA1-mediated localization of Rad51 at DNA damage and D-loop formation. These results are consistent with those of a recent study, which found that prexasertib, another selective CHK1 inhibitor, suppressed Rad51 and BRCA1 expression in triple-negative breast cancer cells by promoting ubiquitin-mediated proteasome degradation, but RNAi-induced CHK1 downregulation only affected the focus-forming capacity of Rad51 (45). However, the detailed mechanism by which CCT245737 inhibits Rad51 and BRCA1 expression in CML cells and whether these effects depend on the timing of drug administration remain to be determined in subsequent experiments. Based on the CCK-8 and western blot results in K562-IM cells, we speculated that imatinib (IM) may force K562 cells into a stress state which helped cells to be more resistant to other pharmacological stimulation. Therefore, a more in-depth comparison of the pharmacological combination of CCT245737 and VP16 between K562-WT and K562-IM cells in terms of DNA damage repair would also be investigated in our future research.

In addition to the critical role of CHK1 in the cell cycle and DNA damage repair pathway, a recent study revealed that a CHK1 inhibitor can also trigger Bcr-Abl protein degradation in CML (46). This is another potential mechanism by which targeting CHK1 could functionally treat CML.

In conclusion, CHK1 is a promising target for the development of CML therapies. CHK1 inhibition can efficiently impair DSB-mediated cell cycle arrest and the HR repair pathway. Therefore, the lack of time and capacity to repair DNA damage after CHK1 inhibition will induce greater accumulation of DNA damage and increase apoptosis, resulting in reductions in proliferation and colony formation. Furthermore, CCT245737 is potentially an ideal candidate CHK1 inhibitor that demonstrates dramatic and specific synergism with VP16 in both wild-type and IM-treated K562 cells. These results provide convincing evidence to promote this strategy in future clinical regimens for CML or even TKI-resistant CML.

\section{Acknowledgements}

Not applicable.

\section{Funding}

This present work was supported by the Ministry of Science and Technology of China (grant no. 2016YFE0107200), the National Major Scientific and Technological Special Project for 'Significant New Drugs Development' (2018ZX09201002-005), the National Natural Science Foundation of China (31471029, $31671055,81461138037)$ and Xu Jun's expert work station (2017IC025).

\section{Availability of data and materials}

The datasets used and/or analyzed in the current study are available from the corresponding author on reasonable request.

\section{Authors' contributions}

$\mathrm{ZF}$, the first author of this article, designed the research, collected the samples, performed the experiments, analyzed 
the data and wrote the manuscript. HL, JZ, FW, WZ, JW, SL, QL, YX, GW, AL and JX participated in sample collection, data collection and analysis. All authors read and approved the manuscript and agree to be accountable for all aspects of the research in ensuring that the accuracy or integrity of any part of the work are appropriately investigated and resolved.

\section{Ethics approval and consent to participate}

Not applicable.

\section{Patient consent for publication}

Not applicable.

\section{Competing interests}

The authors declare that there are no competing interests.

\section{References}

1. Bhamidipati PK, Kantarjian H, Cortes J, Cornelison AM and Jabbour E: Management of imatinib-resistant patients with chronic myeloid leukemia. Ther Adv Hematol 4: 103-117, 2013.

2. Kaleem B, Shahab S, Ahmed N and Shamsi TS: Chronic myeloid leukemia-prognostic value of mutations. Asian Pac J Cancer Prev 16: 7415-7423, 2015

3. Valent P, Hadzijusufovic E, Schernthaner GH, Wolf D, Rea D and le Coutre P: Vascular safety issues in CML patients treated with BCR/ABL1 kinase inhibitors. Blood 125: 901-906, 2015.

4. Experts in Chronic Myeloid Leukemia: The price of drugs for chronic myeloid leukemia (CML) is a reflection of the unsustainable prices of cancer drugs: From the perspective of a large group of CML experts. Blood 121: 4439-4442, 2013.

5. Dillon MT, Good JS and Harrington KJ: Selective targeting of the $\mathrm{G}_{2} / \mathrm{M}$ cell cycle checkpoint to improve the therapeutic index of radiotherapy. Clin Oncol (R Coll Radiol) 26: 257-265, 2014.

6. Del Nagro CJ, Choi J, Xiao Y, Rangell L, Mohan S, Pandita A, Zha J, Jackson PK and O'Brien T: Chk1 inhibition in p53-deficient cell lines drives rapid chromosome fragmentation followed by caspase-independent cell death. Cell Cycle 13: 303-314, 2014.

7. Cimprich KA and Cortez D: ATR: An essential regulator of genome integrity. Nat Rev Mol Cell Biol 9: 616-627, 2008.

8. Yazinski SA and Zou L: Functions, regulation, and therapeutic implications of the ATR checkpoint pathway. Annu Rev Genet 50: 155-173, 2016.

9. Jazayeri A, Falck J, Lukas C, Bartek J, Smith GC, Lukas J and Jackson SP: ATM- and cell cycledependent regulation of ATR in response to DNA double-strand breaks. Nat Cell Biol 8: 37-45, 2006.

10. Sarmento LM, Póvoa V, Nascimento R, Real G, Antunes I, Martins LR, Moita C, Alves PM, Abecasis M, Moita LF, et al: CHK1 overexpression in T-cell acute lymphoblastic leukemia is essential for proliferation and survival by preventing excessive replication stress. Oncogene 34: 2978-2990, 2015.

11. Walworth N, Davey S and Beach D: Fission yeast chk1 protein kinase links the rad checkpoint pathway to cdc2. Nature 363: 368-371, 1993

12. Bartek $\mathbf{J}$ and Lukas $\mathbf{J}:$ Chk1 and Chk2 kinases in checkpoint control and cancer. Cancer Cell 3: 421-429, 2003.

13. Zhang Y and Hunter T: Roles of Chk1 in cell biology and cancer therapy. Int J Cancer 134: 1013-1023, 2014.

14. Patil M, Pabla N and Dong Z: Checkpoint kinase 1 in DNA damage response and cell cycle regulation. Cell Mol Life Sci 70: 4009-4021, 2013

15. Maya-Mendoza A, Petermann E, Gillespie DA, Caldecott KW and Jackson DA: Chk1 regulates the density of active replication origins during the vertebrate S phase. EMBO J 26: 2719-2731, 2007.

16. Tang J, Erikson RL and Liu X: Checkpoint kinase 1 (Chk1) is required for mitotic progression through negative regulation of polo-like kinase 1 (Plk1). Proc Natl Acad Sci USA 103: 11964-11969, 2006.
17. Sorensen CS, Hansen LT, Dziegielewski J, Syljuåsen RG, Lundin C, Bartek J and Helleday T: The cell-cycle checkpoint kinase Chk1 is required for mammalian homologous recombination repair. Nat Cell Biol 7: 195-201, 2005.

18. Wang X, Kennedy RD, Ray K, Stuckert P, Ellenberger T and D'Andrea AD: Chk1-mediated phosphorylation of FANCE is required for the Fanconi anemia/BRCA pathway. Mol Cell Biol 27: 3098-3108, 2007.

19. Walton MI, Eve PD, Hayes A, Valenti MR, De Haven Brandon AK, Box G, Hallsworth A, Smith EL, Boxall KJ, Lainchbury M, et al: CCT244747 is a novel potent and selective CHK1 inhibitor with oral efficacy alone and in combination with genotoxic anticancer drugs. Clin Cancer Res 18: 5650-5661, 2012.

20. Calvo E, Braiteh F, Von Hoff D, McWilliams R, Becerra C, Galsky MD, Jameson G, Lin J, McKane S, Wickremsinhe ER, et al: Phase I study of CHK1 inhibitor LY2603618 in combination with gemcitabine in patients with solid tumors. Oncology 91: 251-260, 2016.

21. Laquente B, Lopez-Martin J, Richards D, Illerhaus G, Chang DZ, Kim G, Stella P, Richel D, Szcylik C, Cascinu S, et al: A phase II study to evaluate LY2603618 in combination with gemcitabine in pancreatic cancer patients. BMC Cancer 17: 137, 2017

22. Yin Y, Shen Q, Zhang P, Tao R, Chang W, Li R, Xie G, Liu W, Zhang L, Kapoor P, et al: Chk1 inhibition potentiates the therapeutic efficacy of PARP inhibitor BMN673 in gastric cancer. Am J Cancer Res 7: 473-483, 2017.

23. Italiano A, Infante JR, Shapiro GI, Moore KN, LoRusso PM, Hamilton E, Cousin S, Toulmonde M, Postel-Vinay S, Tolaney S, et al: Phase I study of the checkpoint kinase 1 inhibitor GDC-0575 in combination with gemcitabine in patients with refractory solid tumors. Ann Oncol 29: 1304-1311, 2018.

24. Restelli V, Lupi M, Vagni M, Chila R, Bertoni F, Damia G and Carrassa L: Combining ibrutinib with Chk1 inhibitors synergistically targets mantle cell lymphoma cell lines. Target Oncol 13: 235-245, 2018.

25. Booth L, Roberts J, Poklepovic A and Dent P: The CHK1 inhibitor SRA737 synergizes with PARP1 inhibitors to kill carcinoma cells. Cancer Biol Ther 19: 786-796, 2018.

26. Walton MI, Eve PD, Hayes A, Henley AT, Valenti MR, De Haven Brandon AK, Box G, Boxall KJ, Tall M, Swales K, et al: The clinical development candidate CCT245737 is an orally active CHK1 inhibitor with preclinical activity in RAS mutant NSCLC and Emicro-MYC driven B-cell lymphoma. Oncotarget 7: 2329-2342, 2016.

27. Osborne JD, Matthews TP, McHardy T, Proisy N, Cheung KM, Lainchbury M, Brown N, Walton MI, Eve PD, Boxall KJ, et al: Multiparameter lead optimization to give an oral checkpoint kinase 1 (CHK1) inhibitor clinical candidate: (R)-5-((4-((Morphol in-2-ylmethyl)amino)-5-(trifluoromethyl)pyridin-2-yl)amino)pyr azine-2-carbonitrile (CCT245737). J Med Chem 59: 5221-5237, 2016.

28. Yang M, Tian X, Fan Z, Yu W, Li Z, Zhou J, Zhang W and Liang A: Targeting RAD51 enhances chemosensitivity of adult T-cell leukemia-lymphoma cells by reducing DNA double-strand break repair. Oncol Rep 42: 2426-2434, 2019.

29. Li L, Ye S, Yang M, Yu W, Fan Z, Zhang H, Hu J, Liang A and Zhang W: SIRT1 downregulation enhances chemosensitivity and survival of adult T-cell leukemia-lymphoma cells by reducing DNA double-strand repair. Oncol Rep 34: 2935-2942, 2015.

30. Livak KJ and Schmittgen TD: Analysis of relative gene expression data using real-time quantitative PCR and the 2(-Delta Delta C(T)) method. Methods 25: 402-408, 2001.

31. Chou TC: Theoretical basis, experimental design, and computerized simulation of synergism and antagonism in drug combination studies. Pharmacol Rev 58: 621-681, 2006.

32. Tirrò E, Massimino M, Romano C, Pennisi MS, Stella S, Vitale SR, Fidilio A, Manzella L, Parrinello NL, Stagno F, et al: Chk1 inhibition restores inotuzumab ozogamicin citotoxicity in CD22-positive cells expressing mutant p53. Front Oncol 9: 57, 2019.

33. Parsels LA, Qian Y, Tanska DM, Gross M, Zhao L, Hassan MC, Arumugarajah S, Parsels JD, Hylander-Gans L, Simeone DM, et al: Assessment of chk1 phosphorylation as a pharmacodynamic biomarker of chk1 inhibition. Clin Cancer Res 17: 3706-3715, 2011.

34. Morimoto S, Tsuda M, Bunch H, Sasanuma H, Austin C and Takeda S: Type II DNA topoisomerases cause spontaneous double-strand breaks in genomic DNA. Genes (Basel) 10: 868, 2019. 
35. Schonn I, Hennesen J and Dartsch DC: Cellular responses to etoposide: Cell death despite cell cycle arrest and repair of DNA damage. Apoptosis 15: 162-172, 2010.

36. Shibata A and Jeggo PA: DNA double-strand break repair in a cellular context. Clin Oncol (R Coll Radiol) 26: 243-249, 2014

37. Chang HHY, Pannunzio NR, Adachi N and Lieber MR: Non-homologous DNA end joining and alternative pathways to double-strand break repair. Nat Rev Mol Cell Biol 18: 495-506, 2017.

38. Savage KI, Gorski JJ, Barros EM, Irwin GW, Manti L, Powell AJ, Pellagatti A, Lukashchuk N, McCance DJ, McCluggage WG, et al: Identification of a BRCA1-mRNA splicing complex required for efficient DNA repair and maintenance of genomic stability. Mol Cell 54: 445-459, 2014

39. Roy R, Chun J and Powell SN: BRCA1 and BRCA2: Different roles in a common pathway of genome protection. Nat Rev Cancer 12: 68-78, 2011.

40. Moynahan ME, Chiu JW, Koller BH and Jasin M: Brca1 controls homology-directed DNA repair. Mol Cell 4: 511-518, 1999.

41. Caestecker KW and Van de Walle GR: The role of BRCA1 in DNA double-strand repair: Past and present. Exp Cell Res 319: 575-587, 2013.
42. Wu LC, Wang ZW, Tsan JT, Spillman MA, Phung A, Xu XL, Yang MC, Hwang LY, Bowcock AM and Baer R: Identification of a RING protein that can interact in vivo with the BRCA1 gene product. Nat Genet 14: 430-440, 1996.

43. Scully R, Chen J, Plug A, Xiao Y, Weaver D, Feunteun J, Ashley T and Livingston DM: Association of BRCA1 with Rad51 in mitotic and meiotic cells. Cell 88: 265-275, 1997.

44. Zhao W, Steinfeld JB, Liang F, Chen X, Maranon DG, Jian Ma C, Kwon Y, Rao T, Wang W, Sheng C, et al: BRCA1-BARD1 promotes RAD51-mediated homologous DNA pairing. Nature 550: 360-365, 2017.

45. Mani C, Jonnalagadda S, Lingareddy J, Awasthi S, Gmeiner WH and Palle K: Prexasertib treatment induces homologous recombination deficiency and synergizes with olaparib in triple-negative breast cancer cells. Breast Cancer Res 21: 104, 2019.

46. Lei H, Jin J, Liu M, Li X, Luo H, Yang L, Xu H and Wu Y: Chk1 inhibitors overcome imatinib resistance in chronic myeloid leukemia cells. Leukemia Res 64: 17-23, 2018.

This work is licensed under a Creative Commons Attribution-NonCommercial-NoDerivatives 4.0 International (CC BY-NC-ND 4.0) License. 